Mexican Journal of Biotechnology 2019, 4(2):10-22

Journal homepage:www.mexjbiotechnol.com

ISSN:2448-6590

ORIGINAL RESEARCH

Characterization of the degradation potential of xenobiotic compounds by the rhizobacteria Azospirillum brasilense

\title{
Caracterización del potencial de degradación de compuestos xenobióticos por la rizobacteria Azospirillum brasilense
}

María Antonia Cruz-Hernández ${ }^{1,2^{*}}$, Juan Miguel Jiménez-Andrade², Alberto Mendoza-Herrera ${ }^{1}$.

${ }^{1}$ Laboratorio Interacción Ambiente Microorganismo, Centro de Biotecnología Genómica-Instituto Politécnico Nacional, Boulevard del Maestro s/n Esq. Elías Piña, Col. Narciso Mendoza, CP. 88710, Cd. Reynosa, Tamaulipas, Mexico.

2Universidad Autónoma de Tamaulipas. Laboratorio de Farmacología. Unidad Académica Multidisciplinaria. Reynosa- Aztlán. Calle 16 y lago de Chapala, Col. Aztlán, CP. 88740, Cd. Reynosa, Tamaulipas, Mexico

${ }^{*}$ Corresponding author

E-mail address: tonitacruz@gmail.com (M. A. Cruz-Hernández).

Article history:

Received: 14 December 2018 / Received in revised form: 15 February 2019 / Accepted: 8 March 2019 / Published online: 1 April 2019.

https://doi.org/10.29267/mxjb.2019.4.2.10

\begin{abstract}
Pollution caused by hydrocarbons represents a risk to the environment. Several studies have focused on finding alternatives to help to prevent hydrocarbon pollution effect. One of them involves the use of native microorganisms capable of persisting on contaminated environments. Therefore, the objective of this work was to analyze the potential of biotypes of Azospirillum brasilense to degrade xenobiotic compounds such as phenanthrene, xylene, toluene and naphthalene. The production of biosurfactants was characterized, and the tolerance to petroleum contaminants was evaluated in vitro, the search for degradation of aromatic compounds related genes was done using the RAST program. The results showed that the strains produced biosurfactants and five of them were selected to test their tolerance to xylene, toluene, phenanthrene and naphthalene. Bacteria show no growth after $216 \mathrm{~h}$ incubation at $30{ }^{\circ} \mathrm{C} .19$ coding sequences were registered
\end{abstract}


regarding to the degradation of aromatic compounds, 11 of them were associated to the metabolism of central aromatic intermediates and 5 were involved in peripheral catabolic pathways whose function is related to degradation pathways of quinate, benzoate, salicylate, gentisate and toluene.

Keywords: degradation, tolerance, pollution.

\section{RESUMEN}

La contaminación ocasionada por hidrocarburos representa un riesgo para el medioambiente. Se han enfocado estudios en la búsqueda de alternativas para contribuir a revertir su efecto. Una de ellas involucra el uso de microorganismos nativos capaces de persistir en ambientes contaminados. Por lo cual el objetivo de este trabajo fue analizar el potencial de biotipos de Azospirillum brasilense para degradar compuestos xenobióticos como fenantreno, xileno, tolueno y naftaleno. Se caracterizó la producción de biosurfactantes, se evaluó in vitro la capacidad de tolerancia a hidrocarburos derivados del petróleo y búsqueda de genes relacionados con la degradación de compuestos aromáticos mediante el programa RAST. Los resultados mostraron que los biotipos produjeron biosurfactantes y se seleccionaron cinco de ellos para realizar ensayos de tolerancia a xileno, tolueno, fenantreno y naftaleno; en los cuales no se observó crecimiento bacteriano después de 216 horas de incubación a $30{ }^{\circ} \mathrm{C}$. Se registraron 19 secuencias codificantes relacionadas con la degradación de compuestos aromáticos; de las cuales 11 están asociadas con el metabolismo de los compuestos intermedios aromáticos centrales y 5 con las vías catabólicas periféricas cuya función se asocia con las rutas de degradación de quinato, benzoato, salicilato, gentisato y tolueno.

Palabras clave: contaminación, degradación, tolerancia.

\section{INTRODUCCIÓN}

Actualmente en nuestro país existen regiones donde la contaminación por hidrocarburos es un problema, a causa principalmente de las actividades de transformación del petróleo, originando daños al ecosistema del suelo, agua y aire (Höhener \& Ponsin, 2013, Fulekar, 2017). A través del uso de microorganismos, se pueden bio-transformar los compuestos tóxicos en productos secundarios de menor toxicidad, o incluso promover la mineralización completa de los hidrocarburos totales del petróleo (Maldonado-Chávez et al., 2010, Gouma et al., 2014, Abbasian et al., 2015, Zhao et al., 2018). El uso de bacterias se ha preferido debido al amplio espectro de hidrocarburos que pueden degradar (Wolicka et al., 2009, Abbasian et al., 2015). Las bacterias del género Azospirillum, se han estudiado ampliamente por su capacidad de promover el crecimiento bacteriano (Steenhoudt y Vanderleyden, 2000, Kaushal \& Wani, 2016, García et al., 2017), y 
se sabe que poseen rutas metabólicas versátiles que emplean carbono y nitrógeno; lo que les ha permitido establecerse en ambientes competitivos donde predominan la desecación y la limitación de nutrientes (Rodríguez-Salazar et al., 2009, Pérez-Castañeda et al., 2011). Adicionalmente, existen reportes sobre el aislamiento de cepas de Azospirillium en suelos contaminados con hidrocarburos, así como su capacidad de degradar petróleo crudo (Eckford et al 2002, Muratova et al., 2005, Miranda-Martínez et al., 2007, Covarrubias et al., 2012). Por lo cual el objetivo de este trabajo fue caracterizar el potencial biodegradador de hidrocarburos derivados del petróleo de biotipos de Azospirillum brasilense. aislados del Noreste de Tamaulipas.

\section{MATERIALES Y METODOS}

Para el material biológico se emplearon 17 biotipos de Azospirillum brasilense aislados de la rizosfera de maíz y sorgo del Noreste de Tamaulipas durante los años 2000 a 2002 pertenecientes al Laboratorio Interacción Ambiente Microorganismo del Instituto Politécnico Nacional.

\subsection{Evaluación de la producción de biosurfactantes}

La caracterización de la producción de biosurfactantes por los biotipos bajo estudio, se realizó a través de la evaluación de la actividad hemolítica de acuerdo a la metodología reportada por Carrillo et al. (1996). La síntesis de biosurfactantes de tipo ramnolípidos con el empleo de la técnica reportada por Siegmund \& Wagner (1991) y la obtención del Índice de Emulsificación (E24) de acuerdo a lo reportado por Illori (2005) la cual se midió a los 5 y 10 minutos. Cada una de estas pruebas se realizó por triplicado y utilizando como controles las cepas de Rodococcus ruber y Sphingobium yanoikuyae.

\subsection{Evaluación in vitro de la capacidad de tolerancia a hidrocarburos derivados del petróleo}

La evaluación in vitro de los biotipos seleccionados de la tolerancia a fenantreno, tolueno, naftaleno y xileno, se realizó empleando la metodología reportada por González-Paredes (2013). Se preparó una solución stock de $400 \mathrm{ug} \mathrm{mL}$ de cada hidrocarburo usando como solvente acetona. Las soluciones se mantuvieron en viales color ámbar para prevenir la foto oxidación del hidrocarburo y se almacenaron a $4{ }^{\circ} \mathrm{C}$ para evitar la volatilización del solvente. Se utilizaron matraces de $250 \mathrm{~mL}$ los cuales contenían $30 \mathrm{~mL}$ de medio líquido Luria Bertani, a éstos se les adicionó por separado cada hidrocarburo a una concentración final de $80 \mathrm{ug} \mathrm{mL}$. Estos se mantuvieron abiertos por media hora en la campana de extracción para que el solvente (Diclorometano) se evaporara. Este procedimiento se realizó para los cuatro compuestos en el estudio. Se inoculó una colonia de cada biotipo en medio TY los cuales fueron incubados en agitación (incubadora SHEL-LAB modelo 1545) durante 24 horas con la finalidad de obtener la biomasa bacteriana. Al finalizar se transfirieron a tubos Eppendorf, se centrifugaron, se 
decantó el sobrenadante y se lavó con solución salina estéril, repitiendo el proceso dos veces, se resuspendió la pastilla con solución salina estéril y de este stock se inocularon a una D.O. de \pm 0.05 matraces con $30 \mathrm{~mL}$ de medio de cultivo. Los ensayos fueron realizados por triplicado.

\subsection{Búsqueda de genes en el genoma de Azospirillum brasilense relacionados con la degradación de compuestos aromáticos}

En este caso se utilizó la secuencia del genoma Azospirillum brasilense CBG-497, debido a que ésta es la única cepa de Azospirillum de la colección del Laboratorio Interacción Ambiente Microorganismo del Instituto Politécnico Nacional secuenciada y con anotación completa por lo cual consideramos que la búsqueda de otros genes como los involucrados en los procesos de biorremediación pudieran tener un impacto biotecnológico.

Esta se organizó en archivos individuales, para el cromosoma de aproximadamente $2.9 \mathrm{Mpb}$ y 5 mega-plásmidos: P1 (1.5 Mpb), P2 (0.731 Mpb), P3 (0.488 Mpb) P4 (0.606 Mpb) P6 (148 Kb) en formato FASTA. Cada archivo se cargó de manera individual al servidor RAST (Rapid Annotation using Subsystem Technology), el cual realiza a través de una anotación rápida y automatizada de genomas provenientes de bacterias y arqueas basada en una tecnología de subsistemas; la asignación del posible rol funcional de cada gen ingresado al sistema de acuerdo con su propia matriz de datos.

\section{RESULTADOS}

\subsection{Caracterización de la producción de biosurfactantes}

No obstante que los 17 biotipos probados presentaron crecimiento en el medio Agar base sangre a las 48 horas de incubación, ninguno de ellos presentó beta hemolisis, únicamente la cepa Rodococcus ruber utilizada como control positivo (Tabla 1). Por otro lado, del $100 \%$ de los biotipos de Azospirillum evaluados, el $47 \%$ formó los complejos tensioactivos aniónico y catiónico con el CTAB y el azul de metileno detectados por la presencia de halos de color azul oscuro más intenso, en particular los biotipos BV102 y AZ106. En cuanto a la respuesta de las cepas control positivas Sphingobium yanoikuyae y Rhodococcus ruber ambas presentaron crecimiento, pero solo en $R$. ruber formó el complejo tensioactivo (Tabla 1). En relación al cálculo del Índice de emulsificación, todos los biotipos de Azospirillum brasilense evaluados presentaron actividad emulsificante. A los cinco minutos de incubación se puede apreciar que los biotipos AZ106 y AZ190 presentaron un índice de emulsificación $(E=24)$ en un intervalo de 50 a $59 \%$, un valor mayor que el resto de los biotipos de Azospirillum brasilense. Asimismo, los resultados en las cepas Sphingobium yanoikuyae $S 72$ y Rhodococcus ruber utilizadas como controles positivos fue de $64.83 \%$ y $44.50 \%$ respectivamente. En 
la medición a los 10 minutos, la mayoría ya no presento actividad emulsificante o esta disminuyó drásticamente. En solo cinco biotipos (AZ141, BV174, AZ106, BV180 y BV190) se apreció el índice de emulsificación (E=24) en un rango de 10 a $22 \%$. En la cepa Sphingobium yanoikuyae utilizada como control positivo se observó un índice de emulsión del $64.83 \%$ hasta los 30 min ( $E=24)$ (Tabla 1).

Tabla 1. Evaluación de la síntesis de biosurfactantes por los biotipos de Azospirillum brasilense.

\begin{tabular}{llllc}
\hline $\begin{array}{c}\text { CÓDIGO DEL } \\
\text { BIOTIPO }\end{array}$ & $\begin{array}{c}\text { PRUEBA DE } \\
\text { HEMOLISIS }\end{array}$ & $\begin{array}{c}\text { PRUEBA } \\
\text { MEDIO S-W }\end{array}$ & \multicolumn{2}{c}{$\begin{array}{c}\text { INDICE DE } \\
\text { E } \mathbf{~ m i n}\end{array}$} \\
& & & 27.33 & $\mathbf{1 0}$ \\
\hline AZ108 & - & + & 13.33 & 0.00 \\
BV172 & - & + & 26.66 & 0.00 \\
BV181 & - & + & 0.00 \\
AZ141 & - & + & 43.33 & 22.50 \\
BV174 & - & + & 38.33 & 22.50 \\
AZ197 & - & + & 07.50 & 0.00 \\
CBG 497 & - & + & 28.33 & 0.00 \\
AZ101 & - & + & 25.00 & 0.00 \\
AZ106 & - & + & 52.50 & 9.16 \\
BV102 & - & + & 33.33 & 0.00 \\
AZ105 & - & + & 0.00 & 0.00 \\
BV180 & - & + & 45.83 & 11.66 \\
AZ190 & - & + & 59.16 & 16.66 \\
BV132 & - & + & 22.50 & 0.00 \\
BV144 & - & + & 26.66 & 0.00 \\
BV146 & - & + & 20.00 & 0.00 \\
BV173 & - & - & 15.00 & 0.00 \\
R. ruber & + & - & 44.50 & 0.00 \\
S. yanoikuyae & - & & 64.83 & 64.83 \\
\hline
\end{tabular}

Con base en los resultados obtenidos de estas pruebas, se seleccionaron los biotipos AZ141, BV174, BV180, BV190 y CBG 497, para la evaluación de éstos en la tolerancia a los contaminantes.

\subsection{Evaluación in vitro de la capacidad de tolerar los principales contaminantes del petróleo}

En los resultados de la evaluación de los cinco biotipos de Azospirillum brasilense con cada uno de los contaminantes (xileno, tolueno, fenantreno y naftaleno) a una concentración de $80 \mu \mathrm{g} / \mathrm{mL}$ no se observó crecimiento bacteriano a las 216 horas de incubación a $30^{\circ} \mathrm{C}$.

3.3. Análisis bioinformático del genoma de Azospirillum brasilense CBG-497 en la plataforma de RAST 
El análisis se realizó de manera individual, para el cromosoma y las secuencias de cada uno de los plásmidos. En cuanto a las características de los subsistemas el estudio se enfocó en la búsqueda de genes involucrados en el metabolismo de compuestos aromáticos. Fueron anotados que 19 secuencias codificantes incluidos tanto en el cromosoma como los plásmidos, a excepción de los plásmidos 2 y 6 los cuales no presentaron secuencias codificantes de participar en el metabolismo de compuestos aromáticos. Al menos 11 están asociadas con el metabolismo de los compuestos intermedios aromáticos centrales, mientras que 5 están involucrados en las vías catabólicas periféricas (las cuales convergen en las rutas centrales). Estos se distribuyeron en el genoma de la siguiente manera, para el cromosoma se asignaron seis, para el plásmido 1 seis, plásmido 3 dos, plásmido 4 seis mismas que se pueden observar en la Tabla 2. Los plásmidos 2 y 6 no se incluyen debido a que no contienen genes que codifican para el Metabolismo de compuestos aromáticos.

Tabla 2. Estructura de secuencias codificantes en el genoma de Azospirillum brasilense

Numero de CDS.

Genes relacionados a:

Cromosoma Plásmido 1 Plásmido 3 Plásmido 4

\begin{tabular}{|c|c|c|c|c|}
\hline $\begin{array}{l}\text { Rutas periféricas para el } \\
\text { catabolismo de compuestos } \\
\text { aromáticos }\end{array}$ & 2 & 1 & 0 & 2 \\
\hline $\begin{array}{l}\text { Degradación anaeróbica de } \\
\text { compuestos aromáticos }\end{array}$ & 0 & 0 & 0 & 0 \\
\hline $\begin{array}{l}\text { Metabolismo de compuestos } \\
\text { intermedios aromáticos } \\
\text { centrales }\end{array}$ & 2 & 3 & 2 & 4 \\
\hline $\begin{array}{l}\text { Metabolismo de compuestos } \\
\text { aromáticos no } \\
\text { subcategorizados }\end{array}$ & 2 & 1 & 0 & 0 \\
\hline $\begin{array}{l}\text { Total de Regiones que codifican } \\
\text { para un gen determinado }\end{array}$ & 6 & 6 & 2 & 6 \\
\hline
\end{tabular}

En relación a la función de secuencias codificantes, se observó que están relacionadas con la degradación de quinato, benzoato, salicilato, gentisato y tolueno como se muestra en la Tabla 3. 
Tabla 3. Genes y enzimas localizadas en el genoma de Azospirillum brasilense.

\begin{tabular}{ll}
\hline \multicolumn{1}{c}{ Genes detectados } & \multicolumn{1}{c}{ Acción } \\
\hline Quib (3-dehidroquinato dehidratasa) (EC 4.21.10) & Liasa \\
Ben E2 (Proteína de transporte de Benzoato) & Transportador \\
FAA (Fumarilacetoacetasa hidrolasa) (EC 3.7.1.2) & Hidrolasa \\
FHF (Fumarilacetoacetato hidrolasa) (EC 3.7.1.2) & Hidrolasa \\
PHBT (putativa 4-hidroxibenzoil-CoA tioesterasa) & Hidrolasa \\
CatD (Beta-ketoadipato enol-lactona hidrolasa) (EC 3.1.1.24) & $\begin{array}{l}\text { Hidrolasa } \\
\text { MRML (mandelato racemasa) }\end{array}$ \\
IQOa Isoquinolina 1-oxidoreductasa subunidad alfa & Oxidoreductasa \\
(EC 1.3.99.16) & Oxidoreductasa \\
IQOa Isoquinolina 1-oxidoreductasa subunidad beta & Oxidoreductasa \\
(EC 1.3.99.16) & Oxidoreductasa \\
SalA (Salicilato hidroxilasa) (EC 1.14.13.1) & zinc-independiente \\
TAD (toluenosulfonato de & alcohol \\
dehidrogenasa) & \\
\hline
\end{tabular}

Como se puede observar, las enzimas fueron en su mayoría del tipo hidrolasas y oxido-reductasas, mismas que son las principalmente reportadas en microorganismos que participan en estos procesos.

\section{DISCUSIÓN}

Las plantas y sus bacterias asociadas también juegan un papel importante en la degradación de los compuestos presentes en el suelo y el aire (Pattanayak \& Dhal., 2014). Los organismos individuales son capaces de metabolizar una gama limitada de sustratos de hidrocarburos (Marin et al., 1996). Sin embargo, es importante realizar estudios de cada uno de ellos para obtener información acerca de su comportamiento y características cuando son confrontados a compuestos xenobióticos.

La liberación de biosurfactantes es una de las estrategias utilizadas por los microorganismos para influir en la absorción de hidrocarburos y compuestos hidrofóbicos en general (Johnsen et al. 2005, Chong \& Li, 2017); debido a que la estructura de los biosurfactantes permite solubilizar los hidrocarburos y con esto hacerlos disponibles para la degradación (Nievas et al., 2008, Hmidet et al., 2017). A pesar de obtener crecimiento de cada una de los 17 biotipos de Azospirillum brasilense en medio base sangre, ninguna de ellas presentó beta hemolisis. Existen estudios en los cuales se ha asociado la actividad hemolítica y la producción de biosurfactantes (Plaza et al., 2006, Lamilla et al., 2018). Sin embargo, ésta no es una prueba contundente debido a que, otros autores como Pacwa-Płociniczak et al. (2014) reportaron que una cepa de Pseudomonas sp la cual resultó positiva para la actividad hemolítica fue insuficiente para confirmar la producción de biosurfactantes, debido a que esta cepa posee además de 
biotensioactivos, $\beta$ - hemolisinas, resultan positivas en la actividad hemolítica, por lo cual se requiere de la aplicación de pruebas adicionales. En la prueba con el medio Siegmund-Wagner, el $100 \%$ de los biotipos de Azospirillum brasilense evaluados crecieron, pero solo ocho de ellos resultaron positivos lo cual fue corroborado de manera visible por la presencia de un halo color oscuro debido a la interacción en la que los tensioactivos aniónicos forman un par iónico insoluble con el tensioactivo catiónico del CTAB y el colorante azul de metileno (Siegmun \& Wagner, 1991). Esta técnica surgió como alternativa al uso de agar sangre para la detección de biosurfactantes, ya que cuenta con varias ventajas, como por ejemplo la no represión catabólica de la producción de biosurfactantes, la no ocurrencia de falsos positivos debido a presencia de hemolisinas, o la mayor estabilidad del agar (Tabuchi et al., 2015). Para el caso de los resultados de la evaluación del índice de emulsificación se observó que los 17 biotipos de Azospirillum brasilense presentaron diferencias en la actividad emulsificante de acuerdo al tiempo de evaluación. A los cinco minutos se observó un Índice de Emulsificación (E24) de 45 a 59\% en los biotipos sin embargo en la medición a los 10 minutos podemos observar que la mayoría ya no presento actividad emulsificante o esta disminuyó drásticamente del (E24) de 10 al 20\%. Estos resultados varían dependiendo del microorganismo en estudio, Illori et al. (2005) evaluaron una cepa de Aeromonas spp. aislada de aguas tropicales. Los biosurfactantes producidos por el organismo emulsificaron un rango de hidrocarburos obteniéndose un (E24) del 65\% al utilizar diésel como fuente de carbono y un (E24) de $22 \%$ al utilizar hexano. Obayori et al. (2009) estudiaron el potencial de producción de biosurfactantes en Pseudomonas sp. cepa LP1 y observaron que el valor del Índice de Emulsificación (E24) fue de $80.33 \pm 1.20$ al utilizar aceite como fuente de carbono después de ocho días de incubación.

En relación a los resultados que muestran que los 17 biotipos fueron sensibles a xileno, naftaleno, fenantreno, tolueno, probados en este estudio. Existen pocos trabajos para comparar los resultados obtenidos con Azospirillum brasilense. Barkovsky et al. (1995) evaluaron la capacidad de degradación de fenol y benzoato en 31 cepas de Azospirillum brasilense y Azospirillum lipoferum aisladas de la rizosfera y rizoplano de diferentes plantas. Los resultados mostraron que solo nueve cepas degradaron benzoato y tres degradaron fenol observándose claramente un grado de especificidad entre las cepas de Azospirillum evaluadas. Muratova et al. (2005) evaluaron la tolerancia de 33 cepas de Azospirillum, de diferentes especies incluyendo Azospirillum brasilense en medios de cultivo a los cuales se les adicionó petróleo crudo ligero al 1\% como fuente de carbono. Ellos observaron que dos cepas $A$. lipoferum SR42 y A. brasilense SR80 degradaron petróleo, con un porcentaje de degradación del 57.5 y $56.5 \%$ respectivamente después de 14 días de incubación.

En los resultados en cuanto a la búsqueda de genes relacionados con la degradación de compuestos aromáticos, las enzimas oxidorreductasas e hidrolasas fueron las más representativas. Chandrakant \& Shwetha (2011), Kadri et al. (2017) reportaron que la detoxificación de compuestos orgánicos tóxicos utilizado por bacterias y hongos es a través de oxidorreductasas los cuales 
participan activamente en el proceso de biorremediación. En este trabajo se reportaron genes y enzimas como 3-Dehidroquinato deshidratasa, fumarilacetoacetasa hidrolasa, beta-ketoadipato enol-lactona hidrolasa, Salicilato hidroxilasa y una proteína de transporte de benzoato lo cual concuerda con lo reportado por Wisniewski-Dyé et al. (2012). En dicha investigación se realizaron análisis de genómica comparativa analizando el genoma de las cepas Azospirillum brasilense Sp245, Azospirillum lipoferum 4B, Azospirillum sp. B510 y Azospirillum brasilense CBG 497. Estos autores reportaron que la degradación de un amplio espectro de compuestos aromáticos y xenobióticos se basa en dos intermediaros centrales: catecol y protocatecuato. Las cepas A. lipoferum 4B y Azospirillum poseen enzimas que permiten la conversión de benzoato y 4-hidroxibenzoato en protocatecuato. Además, Azospirillum sp. B510 puede convertir benzoato en catecol, para el caso de la cepa CBG 497 se encontró el gen Ben E2 que codifica para una proteína de transporte de benzoato. Las cepas de Azospirillum brasilense parecen ser las menos versátiles con solo una vía de degradación de protocatecuato, encontrándose en la cepa CBG 497 el gen Quib que codifica para la enzima 3- dehidroquinato deshidratasa involucrada en esa vía.

Los biotipos de Azospirillum brasilense evaluados presentaron actividad emulsificante, sin embargo, su crecimiento fue inhibido en los medios de cultivo inoculados con los diferentes hidrocarburos. La cepa CBG497 aislada de la rizosfera de gramíneas de esta región, tiene genes relacionados con la degradación de compuestos aromáticos en su genoma, lo cual le podría conferir su adaptación en suelos contaminados con hidrocarburos. Es importante mencionar que éste es el primer estudio que involucra biotipos del género Azospirillum en cuanto a su evaluación del potencial de tolerancia a hidrocarburos, ya que los estudios han sido realizados principalmente en el estudio en sus características como bacterias asociativas que estimulan el crecimiento y el desarrollo de su planta hospedera.

\section{AGRADECIMIENTOS}

Agradecemos al Dr. Alberto Mendoza Herrera (in memoriam), quien fundó la línea de investigación sobre Azospirillum brasilense en el Centro de Biotecnología Genómica, Instituto Politécnico Nacional.

\section{CONFLICTO DE INTERESES}

Los autores declaran que no existe conflicto de intereses. 


\section{REFERENCIAS}

Abbasian F., Lockington R., Mallavarapu M. \& Naidu R. 2015. A Comprehensive Review of Aliphatic Hydrocarbon Biodegradation by Bacteria. Applied Biochemistry and Biotechnology. 176:670-699.

Barkovskii A. L., Korshunova V. E. \& Pozdnyacova L. I. 1995. Catabolism of phenol and benzoate by Azospirillum strains Applied Soil Ecology 2:17-24

Carrillo PG., Mardaraz C., Pitta-Alvarez. \& SJ Giulietti AM. 1996. Isolation and selection of biosurfactant-producing bacteria. World Journal Microbiology and Biotechnology. 12:82-84.

Chandrakant S. K. \& Shwetha S. R. 2011. Role of Microbial Enzymes in the Bioremediation of Pollutants: A Review. Enzyme Research. Article ID 805187, 11 pages.

Chong H \& Li Q. 2017. Microbial production of rhamnolipids: opportunities, challenges and strategies. Microbial Cell Factories. 16:137.

Covarrubias SA., LE de-Bashan., M Moreno. \& Y Bashan. 2012. Alginate beads provide a beneficial physical barrier against native microorganisms in wastewater treated with immobilized bacteria and microalgae. Applied Environmental Microbiology 93, 2669-2680.

Eckford R., Fred D., Cook D., Aislabie J. \& J Foght. 2002. Free-Living Heterotrophic Nitrogen-Fixing Bacteria Isolated from Fuel-Contaminated Antarctic Soils. Applied and Environmental Microbiology p. 5181-5185

Fulekar M. 2017. Microbial degradation of petrochemical waste-polycyclic aromatic hydrocarbons. Bioresources and Bioprocessing. 4:28.

García J.E., Maroniche G., Creus C., Suárez-Rodríguez R., Ramírez-Trujillo J.A., \& Groppa M.D. 2017. In vitro PGPR properties and osmotic tolerance of different Azospirillum native strains and their effects on growth of maize under drought stress. Microbiological Research 202. 21-29

González-Paredes Y., Alarcón A., Ferrera-Cerrato R., Almaraz J.J., MartínezRomero E., Cruz-Sánchez J.S., Mendoza-López M. R. \& Ormeno-Orrillo E. 2013. Tolerance, growth and degradation of phenanthrene and benzo[a]pyrene by Rhizobium tropici CIAT 899 in liquid culture medium. Applied Soil Ecology. 63:105111.

Gouma S., Fragoeiro S., Bastos A.C. \& Magan N. 2014. Bacterial and Fungal Bioremediation Strategies. Microbial Biodegradation and Bioremediation 301-323. 
Hmidet N., Ayed H., Jacques P. \& Nasri M. 2017. Enhancement of Surfactin and Fengycin Production by Bacillus mojavensis A21: Application for Diesel Biodegradation. Volume 2017, ID 5893123

Höhener P. \& Ponsin V. 2013. In situ vadose zone bioremediation. Environmental biotechnology. Current Opinion in Biotechnology. 27: 1-7.

Ilori M.O., Amobi C.J. \& Odocha A.C. 2005. Factors affecting biosurfactant production by oil degrading Aeromonas sp., isolated from a tropical environment. Chemosphere 61:985-992.

Johnsen A.R., Wick L.Y., Harms H. 2005. Principles of microbial PAH-degradation in soil. Environmental Pollution. Jan;133(1):71-84.

Kadri T., Rouissi T., Kaur-Brar S., Cledon M., Sarma S. \& Verma M. 2017. Biodegradation of polycyclic aromatic hydrocarbons (PAHs) by fungal enzymes. A review. Journal of Environmental Sciences (China). Jan;51:52-74. doi: 10.1016/j.jes.2016.08.023. E pub 2016 Oct 3.

Kaushal M. \& Wani S.P. 2016. Plant-growth-promoting rhizobacteria: drought stress alleviators to ameliorate crop production in drylands. Annals of Microbiology 66:35-42.

Lamilla C., Braga D., Castro R., Guimarães C. V. A., de Castilho L., Freire D. \& Barrientos L. 2018. Streptomyces luridus So3.2 from Antarctic soil as a novel producer of compounds with bioemulsification potential. PLoS ONE 13(4): e0196054.

Marin M.A., Pedrogosa A. \& Laborda F.1996. Emulsifier production and microscopical study of emulsions and biofilms from by the hydrocarbon utilizing bacteria Acinetobacter calcoaceticus. MM5. Applied Microbiology and Biotechnology 44:660-667. doi:10.1007/BF00172500.

Maldonado-Chávez E., Rivera-Cruz M.C., Izquierdo-Reyes F. \& Palma-López D.J. 2010. Effects of rhizosphere, microorganisms and fertilization on bioremediation and phytoremediation of soils with new and weathered crude oil. Publicaciones/ciencia 26(2):121-136.

Miranda-Martínez M., Delgadillo-Martínez R. J., Alarcón A. \& Ferrera-Cerrato R. 2007. Degradación de fenantreno por microorganismos en la rizósfera del pasto alemán Terra. Latinoamericana [en linea]

Muratova A.Y., Turkovskaya O. V., Antonyuk L. P., Makarov O. E., Pozdnyakova L. I. \& Ignatov V. V. 2005. Oil-Oxidizing Potential of Associative Rhizobacteria of the Genus Azospirillum Microbiology, Vol. 74(2) pp. 210-215. Translated from 
Mikrobiologiya, Vol. 74, No. 2, 2005, pp. 248-254. Original Russian Text Copyright (C) 2005 by Muratova, Turkovskaya, Antonyuk, Makarov, Pozdnyakova, Ignatov

Nievas M., Commendatore M., Estevas J. \& Bucalá V. 2008. Biodegradation pattern of hydrocarbons from a fuel oil-type complex residue by an emulsifierproducing microbial consortium. Journal of Hazardous Materials. 154(1-3): 96-104.

Obayori O.S., Ilori M.O., Adebusoye S.A., Oyetibo G.O., Omotayo A.E. \& Amund O.O. 2009. Degradation of hydrocarbons and biosurfactant production by Pseudomonas sp. strain LP1. World Journal of Microbiology and Biotechnology 25:1615-1623. DOI 10.1007/s11274-009-0053-z

Pacwa-Płociniczak M., Płaza G. A., Poliwoda A. \& Piotrowska-Seget Z. 2014. Characterization of hydrocarbon-degrading and biosurfactant-producing Pseudomonas sp. P-1 strain as a potential tool for bioremediation of petroleumcontaminated soil. Environmental Science and Pollution Research. 21:9385-9395

Pattanayak B. \& Dhal N. K. 2014. Biotechnological potential of plant microbe interaction with enhanced phytoremediation. Journal of Environmental Research and Development Vol. 8 No. 3A, January-March.

Pérez-Castañeda L.M., Cruz-Hernández M.A. \& Mendoza-Herrera A. 2011. Variabilidad genética de aislamientos no-típicos de Azospirillum brasilense por análisis PCR-RFLP del ADN 16S ribosomal. PHYTON ISSN 00319457 80: 27-34

Plaza G., Zjawiony I. \& Banat I. 2006. Use of different methods for detection of thermophilic biosurfactant-producing bacteria from hydrocarbon-contaminated and bioremediated soils. Journal of Petroleum Science and Engineering. 50: 71-77.

Rodríguez-Salazar J., Suárez R., Caballero-Mellado J., Iturriaga G. 2009. Trehalose accumulation in Azospirillum brasilense improves drought tolerance and biomass in maize plants. FEMS Microbiology Letters. 296(1) 52-59.

Siegmund I. \& Wagner F. 1991. New method for detecting rhamnolipids excreted by Pseudomonas species during growth on mineral agar. Biotechnology Techniques. 5:265-268.

Steenhoudt O. \& Vanderleyden J. 2000. Azospirillum, a free living bacterium closely associated with grasses: genetic, biochemical and ecological aspects. FEMS Microbiology Reviews 24:487-506.

Tabuchi T., Martínez D., Hospinal M., Merino F., Gutiérrez S. 2015. Optimization and modification of the method for detection of rhamnolipids. Revista Peruana de Biologia. Vol.22 no.2 Lima ago. 
Wisniewski-Dyé F., Lozano L., Acosta-Cruz E., Borland S., Drogue B., PrigentCombaret C., Rouy Z., Barbe V., Mendoza Herrera A., González V. \& Mavingui P. 2012. Genome Sequence of Azospirillum brasilense CBG497 and Comparative Analyses of Azospirillum Core and Accessory Genomes provide Insight into Niche Adaptation. Genes 2012(3): 576-602.

Wolicka D., Suszek A. \& Borkowski A. 2009. Application of aerobic microorganisms in bioremediation in situ of soil contaminated by petroleum products. Bioresource Technology. 100(13) 3221-3227.

Zhao Ma., Liu L., Dick R., Li H., Shen D., Gao Y., Waigi M. \& Ling W. 2018. Rhamnolipid influences biosorption and biodegradation of phenanthrene by phenanthrene-degrading strain Pseudomonas sp. Ph6. Environmental Pollution. 240: 359-367. 